\title{
SAÚDE E ATIVIDADES DE LAZER DE JOVENS NO ENSINO MÉDIO
}

\author{
HEALTH AND LEISURE ACTIVITIES OF YOUNG PEOPLE IN HIGH SCHOOL
}

\author{
Rute Estanislava Tolocka ${ }^{\mathrm{a}^{*}}$, Eduardo Pereira Ramos ${ }^{\mathrm{b}^{* *}}$, Luis Paulo Liberato Peruchi ${ }^{\mathrm{c}^{*}}$
}

tkivas05@yahoo.com.bra , nupemlab@gmail.com ${ }^{\mathrm{b}}$, luispaulo_liberato@outlook.com ${ }^{c}$

UNIMEP - Faculdade de Ciências da Saúde *, IFSUL DE MINAS - Instituto Federal do Sul de Minas*

Data de recebimento do artigo: 21/01/2019

Data de aceite do artigo: 25/02/2019

\section{RESUMO}

A literatura indica que atividades de lazer auxiliam na prevenção de patologias, porém, esta relação entre jovens ainda não está bem estudada. Pouco se sabe sobre o nível de saúde de jovens que participam de programas de esporte e lazer e o sedentarismo tem aumentado entre os jovens. O objetivo deste estudo foi verificar a participação de jovens secundaristas em um Programa de Esporte e Lazer (PEL) e doenças relatadas; 350 estudantes de uma escola técnica de segundo grau participaram do estudo, respondendo a um questionário; entre os jovens participantes do PEL 35.5\% relataram ter doenças e este índice chegou a 50.9\%. entre os que não participam do PEL. Foi encontrada correlação estatistica significante entre a participação no programa e não declarar ter doenças. Mais estudos são necessários para diminuir a barreira para as práticas de lazer físico desportivo entre os jovens, especialmente os que estão acometidos por doenças.

Palavras-chave: Atividades de lazer, juventude, doença, educação.

\section{ABSTRACT}

The literature indicate that leisure activities promote well-being but the relationship between health and leisure among youths is not well studied yet. Sedentary lifestyle has increased among the young people. The aim of this study was to analyse the participation of youth in a leisure program and the presence of diseases; 350 students from a technical and high school took part in the study by answering a questionnaire about their participation in the leisure program and diseases they may have. Among the young that took part in the PEL 35.5\% related some disease and among those who did not take part in such program this index reached 50.9\%. It was found significant statistic correlation between to take part in the program and do not declare to have diseases. More studies are needed to diminish barrier for practice of leisure physical activities among young people.

Key-Words: Leisure activities, youth, disease, education 


\section{Introdução}

O conceito de saúde adotado pela Organização Mundial de Saúde, desde sua fundação em 1946, ainda vigente, apresenta saúde como "situação de perfeito bem-estar físico, mental e social e não meramente a ausência de doença ou en-

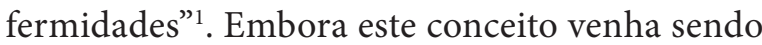
discutido quanto à utopia do perfeito bem-estar e a natureza do ser humano ${ }^{2}$ é notório que consistiu em um avanço sobre a dicotomia entre corpo e mente ${ }^{3}$ e trouxe à luz elementos sociais constitutivos da saúde.

$\mathrm{Na}$ Constituição Brasileira a saúde é tida como direito social e dever do Estado ${ }^{4}$. Determinantes sociais de saúde se relacionam com as condições de vida e trabalho dos indivíduos e de grupos da população. Entre estes determinantes encontram-se acesso à alimentação saudável e ao lazer ${ }^{5}$.

O lazer, apresentado como uma possibilidade de trazer bem-estar aos indivíduos, é necessário para recuperar o trabalhador para o trabalho ${ }^{6} \mathrm{e}$ ocorre pode propiciar repouso, divertimento, atividades sociais ou aprimoramento pessoal"7 ou ainda trazer a possibilidade de escolha do que se pretende fazer, dentro do tempo livre de obrigações. Seu principal benefício é o prazer que o momento é capaz de proporcionar, gerando, divertimento, descanso e desenvolvimento pessoal e social ${ }^{8}$, contribuindo assim, como o bem-estar do indivíduo.

Entre os conteúdos do lazer encontram-se os denominados físico-esportivos ${ }^{9}$ que mestão ligados a práticas que podem levar a um estilo de vida ativo, prevenindo o sedentarismo e patologias associadas, tais como Doenças Crônicas Não Transmissíveis (DCNT) que estão entre as principais causas de mortes e morbidades no Brasil ${ }^{10} \mathrm{e}$ juntas atingem 38\% de jovens na faixa etária de 18 a 24 $\operatorname{anos}^{11}$. Entre os principais fatores de risco destas ocorrências estão o tabagismo, excesso de peso e obesidade, alimentação inadequada, inatividade física e alcoolismo, além de referências aos diagnósticos médicos de diabetes e hipertensão ${ }^{12}$.

Entre os principais benefícios da atividade física no combate a estas doenças estão: redução da adiposidade corporal, regressão da pressão arterial, melhora do perfil lipídico, melhora sensibilidade à insulina, aumento do gasto energético, aumento da massa e força muscular e melhora da capacidade cardiorrespiratória ${ }^{10}$.

O Ministério da Saúde incentiva legalmente a prática de atividade física por meio do Vigitel (sistema de Vigilância de Fatores de Risco para DCNT). A prática regular dessas atividades age como coadjuvante na contenção dos fatores de risco, além de promover a melhora da condição psicológica ${ }^{13}$. Os principais fatores de risco são sedentarismo, estresse, colesterol sérico elevado, inatividade física, obesidade, diabetes, nível de gordura no sangue, manutenção e menor índice de pressão arterial em repouso, estudos também mostram que a pratica de atividade física tem influencia favorável nos níveis pressórico, níveis glicêmicos e perfil lipídico ${ }^{14-15}$.

No entanto, estudos relacionaram vida ativa em atividades de esporte e lazer com promoção e manutenção da saúde dos jovens e tem mostrado prejuízos da inatividade física. Um deles, realizado com estudantes do ensino médio de escolas públicas em Pernambuco, relacionou a prática de esporte, dança ou artes marciais com assistir televisão, consumo de álcool e tabaco. Os resultados apontaram para associação significante entre o uso de tabaco e álcool e a inatividade física no lazer e necessidades de estudos para expansão de condutas de saúde ${ }^{10}$.

Outro estudo, realizado em Minas Gerais, corroborou com esses achados e apontou que a maioria dos jovens realizava lazer fisicamente inativo chamando a atenção para necessidade de incentivo aos jovens para participação em atividades de lazer físico-desportivo, como forma de combate ao sedentarismo e patologias associadas ${ }^{16}$.

Um dos estudos realizados pela Pesquisa Nacional de Saúde Escolar (PeNSE), tendo como público alvo estudantes do ensino fundamental, encontrou baixo nível de alimentação saudável e atividade física e predomínio de hábitos não saudáveis como uso de álcool e drogas entre os estudantes ${ }^{17}$.

De outro lado, há entre jovens participantes do ensino médio profissionalizante os que participam de programas de lazer e atividade física, pois a legislação incentiva fortalecimento de atividades socioculturais, porém, pouco se sabe sobre a saúde e o lazer desta parcela da população. Assim, este estudo propõe verificar a relação entre a prática de lazer e doenças referidas por jovens que frequentam o ensino médio. 


\section{Metodologia}

Trata-se de um estudo de campo, do tipo estudo de caso $^{18}$.

O estudo foi realizado em uma instituição de Educação profissionalizante, integrada ao ensino médio, estabelecida no Estado de Minas Gerais. Todos os estudantes que a frequentavam regularmente foram convidados para participar do estudo, dos quais 370 aderiram e assinaram o Termo de Consentimento Livre e Esclarecido [TCLE].

O estudo está de acordo com a declaração de Helsinque, que no Brasil é regida pela resolução do Conselho Nacional de Saúde, CNS 466/2012, foi aprovado pelo Comitê de Ética em Pesquisa da Universidade responsável por meio do Parecer 67/2014 e a instituição concedeu autorização para a realização do mesmo.

A coleta de dados foi feita mediante a um questionário, semiestruturado, com questões referentes à faixa etária, relatos de doenças e participação em atividades de lazer extracurriculares oferecidas no campus.

Os alunos receberam explicações sobre o estudo em uma reunião realizada na própria instituição e em seguida foi apresentado o TCLE. Após o esclarecimento das dúvidas, foi solicitado que os alunos interessados em participar da pesquisa assinassem o TCLE, preenchessem o questionário e o devolvessem.

Os resultados foram analisados com testes estatísticos não paramétricos, por ter dados categóricos, foram utilizados os testes de Qui-quadrado para as análises de associação e o grau da associação através do teste de Coeficiente de Contingência, com nível de significância para $\mathrm{p}<0.05$. Os testes foram realizados no pacote estatístico Statistical Package for the Social Sciences (SPSS ${ }^{\circledast}$ ) versão 20.0 for Windows ${ }^{\oplus}$. Foi feita a análise descritiva com apresentação da frequência relativa das respostas de cada item.

\section{Resultados}

Entre os participantes do estudo, $51.35 \%$ era do gênero masculino; $85.16 \%$ tinha entre $15-17$ anos; $83.2 \%$ dos jovens não referiram patologias e $61.1 \%$ participavam do Programa de Esporte e Lazer (PEL), que ocorre em período extraclasse, como atividade extra não obrigatória. Entre os jovens que participavam do PEL, 35.5\% relataram ter patologias e este índice chegou a 50.9\%. entre os que não participam do PEL. A distribuição destes dados está ilustrada na Tabela 1.

Tabela 1 - Características do grupo estudado

\begin{tabular}{|c|c|c|c|c|c|}
\hline \multicolumn{2}{|c|}{ Característica } & \multirow[t]{2}{*}{$\begin{array}{c}\text { Distribuição } \\
\text { relativa (\%) }\end{array}$} & \multicolumn{2}{|c|}{ Participação no PEL } & \multirow[t]{2}{*}{ Total } \\
\hline & & & SIM & NÃO & \\
\hline \multirow[t]{4}{*}{ Gênero } & Feminino & 46,49 & 46,7 & 53,3 & 100 \\
\hline & Masculino & 51,35 & 30,9 & 69,1 & 100 \\
\hline & Total & 100 & & & \\
\hline & $\begin{array}{l}\text { Abaixo de } 15 \\
\text { anos }\end{array}$ & 0,89 & 0 & 100 & 100 \\
\hline \multirow[t]{3}{*}{ Faixa etária } & 15-17 anos & 85,16 & 41,7 & 58,3 & 100 \\
\hline & $\begin{array}{c}\text { Acima de } 18 \\
\text { anos }\end{array}$ & 13,95 & 23,5 & 76,5 & 100 \\
\hline & Total & 100 & & & \\
\hline \multirow[t]{3}{*}{ Patologias } & Sem relato & 83,2 & 36,5 & 63,5 & 100 \\
\hline & Com relatos & 16,8 & 50,9 & 49,1 & 100 \\
\hline & Total & 100 & & & \\
\hline
\end{tabular}


A participação no PEL foi inversamente proporcional e levemente significativa nos relatos de existência de patologias, com Coeficiente de contingência de Pearson - $\mathrm{CC}=0,109$, e r=0,043), para ao grau de significância $5 \%$, ou seja, jovens que participaram do programa relataram menos patologias que os que não o fizeram.

Foram relatadas os seguintes acometimentos: trombose, alterações nos rins, reumatismo, asma brônquica, bronquite; miopia, doença cardiovascular, hipertensão, sinusite, rinite, enxaqueca, gastrite, lúpus, amidalite, epilepsia, dermatite, hipotireoidismo, hipoglicemia, anemia, alterações posturais (escoliose, lordose e desvio na coluna, dores do joelho); hiperatividade e distúrbio de atenção.

\section{Discussão}

A correlação encontrada entre a participação no PEL e a ausência de patologias, pode ser entendia a partir da premissa de que a prática de atividade física está ligada à prevenção de patologias, pois há evidências epidemiológicas da associação entre atividade física / aptidão física e longevidade, mortalidade e saúde ${ }^{19}$ e que a avaliação da condição de saúde já inclui a prática de atividade física em estudos do IBGE, Ministério da Saúde, dentre outros ${ }^{20}$.

Pode-se considerar também que jovens com patologias tendem a não realizar atividades físico-desportivas, sendo necessário estudos que verifiquem se as práticas de atividade física promovidas em atividades de lazer para jovens incluem ou não adaptações que permitiriam e estimulariam esta prática a partir de protocolos específicos, necessários para prevenir efeitos deletérios ${ }^{21}$. Se pessoas com doenças se afastam da prática de atividades físicas elas perdem os benefícios que tal prática pode trazer, o que pode inclusive complicar o quadro clínico, criando um círculo vicioso de inatividade física que é preciso ser rompido, sendo necessário mais divulgação sobre benefícios da prática de atividade física e necessidades específicas de jovens que tem alguma doença. Vale lembrar que a atividade física age como coadjuvante na contenção de várias doenças, tais como doenças cardíacas ${ }^{22,23}$.

Além disso, a saúde é muito mais ampla que a mera ausência de patologias e é determinada não somente por fatores biológicos e individuais, mas também por dimensões sociais ${ }^{5}$. Para que o lazer possa ser visto como um fator de prevenção e promoção de saúde, não basta que se promovam a prática de atividades físicas, é necessário também que se proporcione bem-estar pelo exercício de liberdade de escolha na realização ou não de atividades que trazem prazer $^{6-8}$, inclusive para que se tenha melhor aderência a esta prática.

O lazer, assim como a saúde, entre outros bens e serviços sociais, é um direito social ${ }^{4}$ capaz de gerar satisfação e prazer através da escolha, inclui determinantes sociais como acesso à cultura, sociabilidade entre outros que resultam na manutenção da saúde ${ }^{5}$ e que devem estar também relacionados com a prática atividades físicas ${ }^{20}$.

Assim, tornar a prática de atividade física prazerosa é uma estratégia importante para prevenção de doenças e deve estar atrelada a busca por qualidade de vida e bem-estar global ${ }^{1}$, o que também implica em mudanças sociais ${ }^{24} \mathrm{e}$ valorização do tempo livre.

\section{Conclusão}

A prática de lazer por alunos do ensino médio integrado a educação profissionalizante está associada à ocorrência de patologias entre os jovens, sendo que entre os que participam de atividades de lazer há menor ocorrência de doenças e isto pode significar também que jovens que possuem doenças se afastam da prática de atividades físicas.

Os benefícios do lazer para a saúde não se restringem às características biológicas como condicionamento físico e um estilo de vida ativa; possibilidades psicosociais precisam tambem ser consideradas, bem como efeitos deleterios da prática do exercício físico e modificações necessárias para possibilitar a prática adequada e atrativa para jovens com patologias.

A diminuição das barreiras que impedem os alunos de participar em tais atividades é um caminho que precisa ser percorrido, no sentido de garantir oportunidades de prática, sendo necessário novos estudos que ampliem o conhecimento sobre prática de lazer físico-desportivo e doenças, para que pessoas com condições adversas possam também dele desfrutar. 


\section{Agradecimentos}

Agradecemos a Coordenadoria de Aperfeiçoamento de Pessoal do Nível Superior (CAPES) ao Fundo de Apoio à Pesquisa da Instituição promotora (FAP), por suporte financeiro ao estudo, bem como à Instituição que o autorizou e a todos que dele participaram.

\section{Fontes de financiamento: CAPES e FAP}

\section{Referências}

1. World Health Organization (WHO). Preamble to the Constitution of WHO as adopted by the International Health Conference. New York: World Health Organization; 1946.

2. Segre M, Ferraz FC. O conceito de saúde. Rev. Saúde Pública. 1997;31(5):538-42.

3. Sá J, Miranda LS. Desconstruindo a definição de saúde. Jornal do Conselho Federal de Medicina. 2004; jul/ago/set:15-16.

4. República Federativa do Brasil (BR). Constituição de 1988. Brasília: Edições Câmara; 2012.

5. Buss PM, Pellegrini Filho, A. A saúde e seus determinantes sociais. PHYSIS: Rev. Saúde Coletiva. 2007;17(1):77-93.

6. Requixa, R. Cadernos de Lazer: Documento 1. São Paulo: SESC; 1976.

7. Parker, S. A sociologia do lazer. Rio de Janeiro: Zahar; 1978.

8. Marcellino, NC. Estudos do Lazer: Uma introdução. Campinas: Autores Associados; 2012.

9. Dumazadier J. Questionamento teórico do lazer. Porto Alegre: CELAR; 1975.

10. Tenório MCM, Barros VGB, Tassitano RM, Bezerra J, Tenório JM, Hallal PC. Atividade Física e comportamento sedentário em adolescentes estudantes do Ensino Médio. Revista Brasileira de Epidemiologia. 2010;13(1):105-7.

11. Portal da saúde. Vigitel. Ministério da Saúde. Obesidade estabiliza no Brasil, mas excesso de peso aumenta; 2014. [acesso em: 30 dez. 2018]. Disponível em: http://portalms.saude.gov.br/noticias/svs/17455-obesidade-estabiliza-no-brasil-mas-excesso-de-peso-aumenta.

12. Brasil. Vigilância de fatores de risco e proteção para doenças crônicas por inquérito telefônico (Vigitel). Ministério de Saúde; 2018. [acesso em: 28 nov. 2018]. Disponível em: http://portalarquivos.saude.gov.br/images/pdf/2017/ abril/17/Vigitel.pdf.
13. Rigatto, AM; Alves, SCC. Exercício e Performance na Obesidade. In Pellegrinotti, I L. Performance Humana saúde e esporte. Ribeirão Preto: Tecmeed; 2004.

14. Pitanga, FJG; et al. Atividade Física no Tempo Livre, porém não Atividade Física no Deslocamento, está Associada com Risco Cardiovascular em Participantes do ELSA-Brasil. Arq Bras Cardiol. 2017;110(1):36-43

15. Lima, GO; Mendes, BM; Kelin, SK; Formentin, CM, Garlipp, DC. Nível de atividade física e risco de desenvolvimento de doenças cardiovasculares em acadêmicos do curso de Educação Física. Revista Brasileira de Prescrição e Fisiologia do Exercício. 2017;11(68):542-9.

16. Maciel MG, Veiga RT. Intenção de mudança de comportamento em adolescentes para a prática de atividades físicas de lazer. Rev. bras. educ. fís. esporte. 2012;26(4):705-16.

17. Reis ACR, Malta DC, Furtado LAC. Desafia para políticas públicas voltadas à adolecência e juventude a partir da Pesquisa Nacional de Saúde e Escola (PeNSE). Ciência \& Saúde Coletiva. 2018;23(9):2879-90.

18. Severino AJ. Metodologia da Pesquisa Científica: revista e atualizada. São Paulo: Cortez; 2012.

19. Pitanga, FJG. Epidemiologia, atividade física e saúde. Rev. Bras. Ciên. e Mov. Brasília 2002; 10(3):49-54.

20. Knuth, AG; Malta, DC; Dumith, SC; Pereira, CA; Moraes Neto, OL; Temporão, GP et al. Prática de atividade física e sedentarismo em brasileiros: resultados da Pesquisa Nacional por Amostra de Domicílios (PNAD). Ciência \& Saúde Coletiva. 2011;16(9):3697705.

21. Coelho, CF; Burini, RC. Atividade física para prevenção e tratamento das doenças crônicas não transmissíveis e da incapacidade funcional. Revista de Nutrição. Pontifícia Universidade Católica de Campinas, Disponível em: http:// hdl.handle.net/11449/13407. Acesso em: 19 jan. 2019.

22. Pitanga, FJG; Matos, SMA; Almeida, MDAC; Barreto, SM; Aquino, EML. Atividade Física no Tempo Livre, porém não Atividade Física no Deslocamento, está Associada com Risco Cardiovascular em Participantes do ELSA-Brasil. Arq Bras Cardiol. 2017;110(1):36-43

23. Lima, GO; Machado, BM; Klein, SK; Formentin, CM; Garlipp, DC. Nível de atividade física e risco de desenvolvimento de doenças cardiovasculares em acadêmicos do curso de educação física. Revista Brasileira de Prescrição e Fisiologia do Exercício. 2017;11(68):542-9.

24. Matiello Junior, E. Bacheladenski, MS. Contribuições do campo crítico do lazer para a promoção da saúde. Revista Ciência \& Saúde Coletiva. 2010;15(5):2569-79. 